\title{
Realization of the tradeoff between internal and external entanglement
}

\author{
Jie Zhu, ${ }^{1,3}$ Meng-Jun Hu, ${ }^{1,3}$ Yue Dai, ${ }^{2,6}$ Yan-Kui Bai, ${ }^{4}$ S. Camalet, ${ }^{5}$ Chengjie Zhang,,${ }^{2,6,}{ }^{*}$ Chuan-Feng Li, ${ }^{1,3}$ \\ Guang-Can Guo, ${ }^{1,3}$ and Yong-Sheng Zhang ${ }^{1,3, \uparrow}$ \\ ${ }^{1}$ Key Laboratory of Quantum Information, University of Science and Technology of China, CAS, Hefei 230026, China \\ ${ }^{2}$ School of Physical Science and Technology, Ningbo University, Ningbo 315211, China \\ ${ }^{3}$ CAS Center for Excellence in Quantum Information and Quantum Physics, Hefei 230026, China \\ ${ }^{4}$ College of Physics and Hebei Key Laboratory of Photophysics Research and Application, Hebei Normal University, \\ Shijiazhuang, Hebei 050024, China \\ ${ }^{5}$ Sorbonne Université, CNRS, Laboratoire de Physique Théorique de la Matière Condensée, LPTMC, F-75005 Paris, France \\ ${ }^{6}$ School of Physical Science and Technology, Soochow University, Suzhou 215006, China
}

(Received 9 January 2019; revised 20 August 2020; accepted 25 August 2020; published 13 October 2020)

\begin{abstract}
We experimentally realize the internal and external entanglement tradeoff, which is a kind of entanglement monogamy relation different from that usually discussed. Using a source of twin photons, we find that the external entanglement in the twin photons and the path-polarization internal entanglement of one photon limit each other. In the extreme case, when the internal state is maximally entangled, the external entanglement must be vanishing, which illustrates entanglement monogamy. Our results of the experiment coincide with the theoretical predictions and therefore provide a direct experimental observation of the internal and external entanglement monogamy relations.
\end{abstract}

DOI: 10.1103/PhysRevResearch.2.043068

\section{INTRODUCTION}

Entanglement monogamy is one of the most fundamental properties for multipartite quantum states, which means that if two qubits, $A$ and $B$, are maximally entangled, then $A$ or $B$ cannot be entangled with the third qubit $C[1,2]$. The quantitative entanglement monogamy inequality was first proved by Coffman, Kundu, and Wootters (CKW) for three-qubit states [3],

$$
C_{A \mid B}^{2}+C_{A \mid C}^{2} \leqslant C_{A \mid B C}^{2},
$$

where $C^{2}$ denotes the squared concurrence for quantifying bipartite entanglement [4]. From Eq. (1), one can easily find that there is a consequent tradeoff between the amount of entanglement shared by qubits $A$ and $B$ and the entanglement shared by qubits $A$ and $C$. For three-qubit pure states, the difference between the right-hand side and the left-hand side of Eq. (1) is defined as the so-called "three-tangle" [3], which is a genuine three-qubit entanglement measure. After the CKW inequality, several entanglement monogamy inequalities [5-41] and even monogamy equalities $[42,43]$ were introduced. Osborne and Verstraete proved the CKW monogamy inequality for $N$-qubit states [5]. In Refs. [6,7], the CKW inequality was generalized

\footnotetext{
*chengjie.zhang@gmail.com

${ }^{\dagger}$ yshzhang@ustc.edu.cn
}

Published by the American Physical Society under the terms of the Creative Commons Attribution 4.0 International license. Further distribution of this work must maintain attribution to the author(s) and the published article's title, journal citation, and DOI. to Gaussian states. Moreover, other entanglement measures, such as the squashed entanglement $[10,11]$, the negativity [12-15], and the squared entanglement of formation [16-18], were also employed to derive the corresponding entanglement monogamy inequalities. On the other hand, many experimental results on entanglement and nonlocality have also been reported $[44,45]$, including entanglement monogamy in experiments [46,47].

Recently, new kinds of monogamy relations have been derived by Camalet [48-51], i.e., internal entanglement (or local quantum resource) and external entanglement have a tradeoff. The usually discussed entanglement monogamy inequalities in Refs. [5-7,11-14,16-18] indicate the tradeoff relation between $E\left(\varrho_{A B}\right)$ and $E\left(\varrho_{A C}\right)$ (or its extension to the $N$-partite case), where $E$ is one kind of entanglement measure and $\varrho_{A B}$ and $\varrho_{A C}$ are reduced density matrices from a three-qubit state. Unlike these previously derived inequalities, Camalet has proposed a different entanglement monogamy inequality [48]. This monogamy relation shows that the local resource can influence the entanglement between subsystems and other external systems. Besides there is no limitation about local resources, which can be entanglement between different degrees, entanglement between different particles of the subsystems, local coherence, and so on. Consider the tripartite quantum state $\varrho_{A_{1} A_{2} B}$ illustrated in Fig. 1, where $A_{1}$ and $A_{2}$ come from the same physical system, but have been encoded in different degrees of freedom, and $B$ is encoded in another physical system. This inequality shows the tradeoff relation between the internal entanglement $\tilde{E}_{A_{1} \mid A_{2}}$ and the external entanglement $E_{A_{1} A_{2} \mid B}$, where $\tilde{E}$ and $E$ are two different but related entanglement measures, and $E_{A_{1} A_{2} \mid B}\left(\tilde{E}_{A_{1} \mid A_{2}}\right)$ denotes the entanglement of $\varrho_{A_{1} A_{2} B}\left(\varrho_{A_{1} A_{2}}\right)$ under the bipartition 


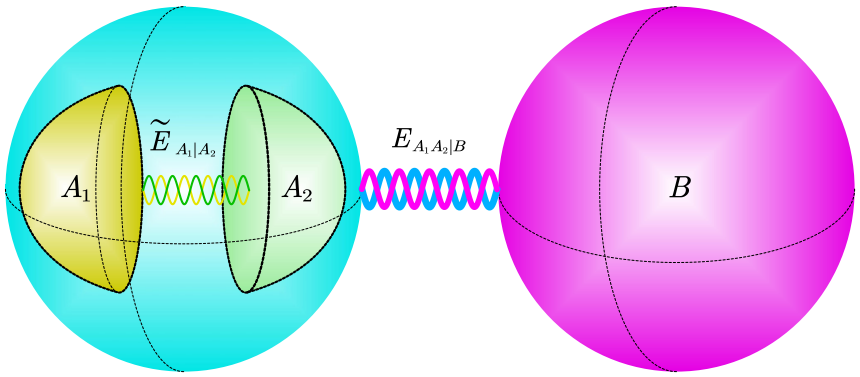

FIG. 1. For the tripartite quantum state $\varrho_{A_{1} A_{2} B}$, the subsystems $A_{1}$ and $A_{2}$ are in the same physical system but they are encoded in different degrees of freedom, and $B$ is encoded in another physical system. $\tilde{E}_{A_{1} \mid A_{2}}$ and $E_{A_{1} A_{2} \mid B}$ represent the internal entanglement between $A_{1}$ and $A_{2}$ and the external entanglement between $A_{1} A_{2}$ and $B$, respectively.

$A_{1} A_{2} \mid B\left(A_{1} \mid A_{2}\right)$. The inequality holds whatever is the underlying physical nature of the internal entanglement. It can be entanglement between two long-distance-separated particles, two short-distance-separated particles, or even 2 degrees of freedom of a single particle. This last kind of internal entanglement is considered here and for precision we refer to it as intraparticle entanglement.

Here we experimentally demonstrate the entanglement monogamy relation between the intraparticle and external entanglement, with a source of twin photons. As shown in Fig. 1, there are two qubits (the polarization qubit $A_{1}$ and the path qubit $A_{2}$ ) encoded in photon $A$, but only one qubit (the polarization qubit $B$ ) is encoded in photon $B$. We provide a direct experimental observation of the tradeoff between the intraparticle entanglement in $A_{1} \mid A_{2}$ and the external entanglement in $A_{1} A_{2} \mid B$.

\section{THEORETICAL FRAMEWORK}

Let us focus on the tripartite state $\varrho_{A_{1} A_{2} B}$, where $A_{1}$ and $A_{2}$ are encoded in the same physical system $A$ by using different degrees of freedom (see Fig. 1). The third party is encoded in system $B$. Camalet's entanglement monogamy inequality is

$$
\tilde{E}_{A_{1} \mid A_{2}}+E_{A_{1} A_{2} \mid B} \leqslant \tilde{E}_{\max },
$$

where $\tilde{E}_{A_{1} \mid A_{2}}$ denotes the intraparticle entanglement measure between $A_{1}$ and $A_{2}, E_{A_{1} A_{2} \mid B}$ is the external entanglement measure between $A_{1} A_{2}$ and $B$, and $\tilde{E}_{\text {max }}$ is the value of $\tilde{E}_{A_{1} \mid A_{2}}$ when $A_{1}$ and $A_{2}$ are maximally entangled [48]. It is worth noting that $\tilde{E}$ and $E$ are strongly related, although they are two different entanglement measures. From the inequality (2), one can see that $E_{A_{1} A_{2} \mid B}$ is also bounded by $\tilde{E}_{\max }$. When the state $\varrho_{A_{1} A_{2} B}$ is pure and the reduced density operator $\varrho_{A_{1} A_{2}}$ is absolutely separable [52,53], the external entanglement $E_{A_{1} A_{2} \mid B}$ is equal to the maximum value $\tilde{E}_{\max }$. On the other hand, when the intraparticle entanglement $\tilde{E}_{A_{1} \mid A_{2}}$ is maximal, the external entanglement $E_{A_{1} A_{2} \mid B}$ must be vanishing.

Inequality (2) holds for any convex measure $\tilde{E}_{A_{1} \mid A_{2}}$ which can thus be, for instance, the entanglement of formation $E_{F}$ [4], the negativity $E_{N}$ [54,55], and the relative entropy of entanglement $E_{R}$ [56]. When we choose the entanglement of formation $E_{F}$ to quantify the intraparticle entanglement between $A_{1}$ and $A_{2}$, the inequality (2) for a general three-qubit pure state $|\psi\rangle_{A_{1} A_{2} B}$ becomes

$$
E_{F}\left(\varrho_{A_{1} A_{2}}\right)+E_{F}^{\prime}\left(|\psi\rangle_{A_{1} A_{2} \mid B}\right) \leqslant 1,
$$

where the intraparticle entanglement $\tilde{E}_{A_{1} \mid A_{2}}$ is $E_{F}\left(\varrho_{A_{1} A_{2}}\right)=$ $H\left(1 / 2+\sqrt{1-C^{2}\left(\varrho_{A_{1} A_{2}}\right)} / 2\right), \quad H$ is the binary entropy $H(x):=-x \log _{2} x-(1-x) \log _{2}(1-x), \quad$ and $\quad C(\varrho)=$ $\max \left\{0, \sigma_{1}-\sigma_{2}-\sigma_{3}-\sigma_{4}\right\}$ is the concurrence of $\varrho$ with $\left\{\sigma_{i}\right\}$ being the square roots of eigenvalues of $\varrho \sigma_{y} \otimes \sigma_{y} \varrho^{*} \sigma_{y} \otimes \sigma_{y}$ in decreasing order [4]. The external entanglement $E_{A_{1} A_{2} \mid B}$ is $E_{F}^{\prime}\left(|\psi\rangle_{A_{1} A_{2} \mid B}\right)$, as defined by

$$
\begin{aligned}
E_{F}^{\prime}\left(|\psi\rangle_{A_{1} A_{2} \mid B}\right) & :=1-\max _{U} E_{F}\left(U \varrho_{A_{1} A_{2}} U^{\dagger}\right) \\
& =1-f\left(\max \left\{0, \lambda_{1}-\lambda_{3}-2 \sqrt{\lambda_{2} \lambda_{4}}\right\}\right),
\end{aligned}
$$

where $U$ denotes the unitary operators of $A, f(x):=$ $H\left(1 / 2+\sqrt{1-x^{2}} / 2\right)$, and $\left\{\lambda_{i}\right\}$ are the eigenvalues of $\varrho_{A_{1} A_{2}}$ in nonascending order [48,53]. In Ref. [53], the maximum entanglement for a given spectrum $\left\{\lambda_{i}\right\}$ measured by the negativity and the relative entropy of entanglement have also been provided. Thus, one can obtain the inequality (2) with the intraparticle entanglement measure being the negativity and the relative entropy of entanglement as well (see Appendix A).

For simplicity, we first consider a class of three-qubit pure states with one parameter $\phi$ as an example:

$$
|\Psi\rangle=\left(\cos \phi|110\rangle+\sin \phi \frac{|01\rangle+|10\rangle}{\sqrt{2}}|1\rangle\right)_{A_{1} A_{2} B} .
$$

Based on Eqs. (3) and (4), one can obtain its intraparticle and external entanglement by using the entanglement of formation, i.e., $E_{F}\left(\varrho_{A_{1} A_{2}}\right)=f\left(\sin ^{2} \phi\right)$ and $E_{F}^{\prime}\left(|\psi\rangle_{A_{1} A_{2} \mid B}\right)=1-$ $f\left(\max \left\{\cos ^{2} \phi, \sin ^{2} \phi\right\}\right)$. Moreover, the states such as

$$
|\Phi\rangle=\left(\frac{1}{\sqrt{2}}|110\rangle+\frac{\cos \theta|01\rangle+\sin \theta|10\rangle}{\sqrt{2}}|1\rangle\right)_{A_{1} A_{2} B}
$$

are also analyzed. For these states, we find $E_{F}\left(\varrho_{A_{1} A_{2}}\right)=$ $f\left(\frac{1}{2} \sin 2 \theta\right)$, which depends on $\theta$, and $E_{F}^{\prime}\left(|\psi\rangle_{A_{1} A_{2} \mid B}\right)=1-$ $f(1 / 2) \approx 0.645$ is a constant. The theoretical results are shown in Fig. 2 as the solid and dotted lines. We can see that all the results are bounded by the green dashed line, i.e., the monogamy inequality (3) always holds. Moreover, the inequality (3) is valid for any finite-dimensional subsystems. However, not all the general allowed region can be reached with three-qubit states. For all the three-qubit pure states, since $\lambda_{1} \geqslant 1 / 2$ and there are at most only two nonzero eigenvalues $\left\{\lambda_{1}, \lambda_{2}\right\}$ for $\varrho_{A_{1} A_{2}}, E_{F}^{\prime}=1-f\left(\max \left\{0, \lambda_{1}\right\}\right) \leqslant$ $1-f(1 / 2) \approx 0.645$, and this bound also holds for all threequbit mixed states. Therefore, the allowed region specific to all three-qubit states is given in Fig. 2, and our experimental points can reach the corresponding borders. The GHZ state is a three-qubit state, so it cannot reach the maximum external entanglement 1 ; it can only reach the maximum 0.645 for three-qubit states.

\section{EXPERIMENTAL REALIZATION}

In order to demonstrate this entanglement monogamy relation, we prepare some quantum states where the quantity 


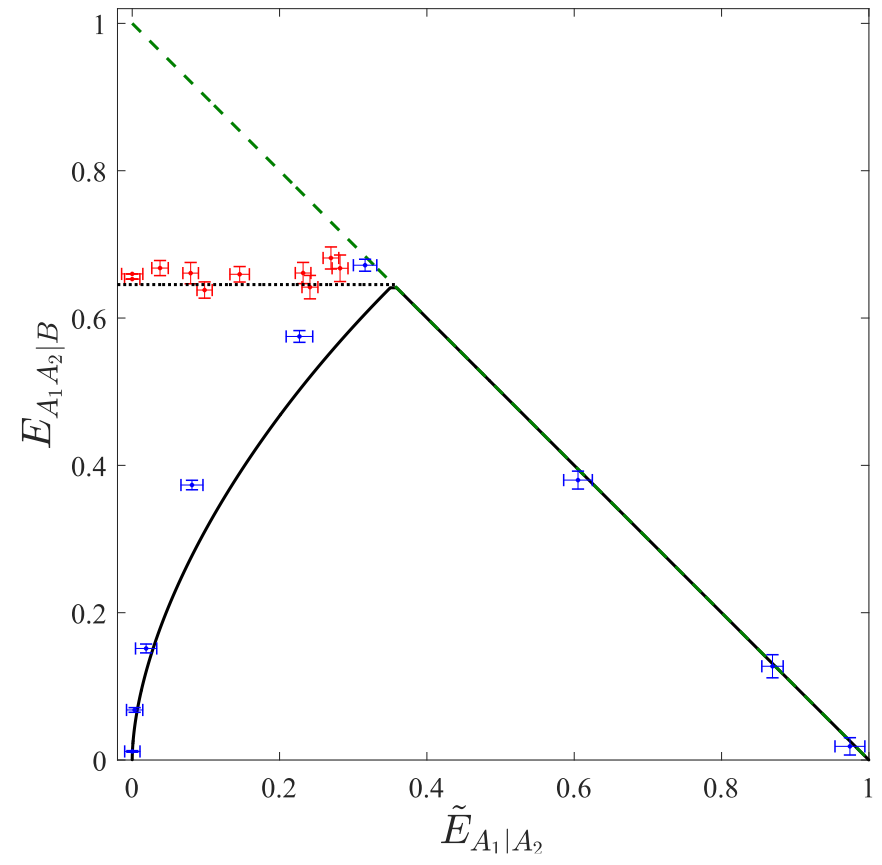

FIG. 2. The intraparticle entanglement $\tilde{E}_{A_{1} \mid A_{2}}$ quantified by $E_{F}\left(\varrho_{A_{1} A_{2}}\right)$ and the external entanglement $E_{A_{1} A_{2} \mid B}$ quantified by $E_{F}^{\prime}\left(|\psi\rangle_{A_{1} A_{2} \mid B}\right)$ are bounded by the green dashed straight line for all tripartite states with finite dimension. Meanwhile, for all the three-qubit states $E_{F}^{\prime}\left(|\psi\rangle_{A_{1} A_{2} \mid B}\right)$ is bounded by the dotted line, i.e., $E_{F}^{\prime}\left(|\psi\rangle_{A_{1} A_{2} \mid B}\right) \leqslant 1-f(1 / 2) \approx 0.645$. The states given by Eqs. (5) and (6) and the corresponding experimental states are shown here. The blue dots correspond to Eq. (5) and the red ones to Eq. (6). All blue dots should be on the solid line and all red ones on the dotted line according to the theoretical predictions but some dots are not. The deviation is from the visibility of interferometers, and the error bars are from the Poissonian distribution of photon counts and the uncertainty of wave plates.

of intraparticle and external entanglement can be controlled. We use the polarization and the path degrees of freedom to produce the target three-qubit states in Eqs. (5) and (6).
As shown in Fig. 3, we introduce three parts of the setup: (i) state preparation, (ii) qubits $A_{1}$ and $A_{2}$ (owned by Alice), and (iii) qubit $B$ (owned by Bob). First, the source of twin photons is realized via a type-I spontaneous parametric down-conversion process in which the crystal is a joint $\beta$-barium borate $(\mathrm{BBO})$ whose size is $8.0 \times 8.0 \times 0.3 \mathrm{~mm}$ and whose optic axis is cut at $\theta_{p m}=29.3^{\circ}$ [57]. The source of the two-qubit entangled state is $|\psi\rangle=\cos \phi|H\rangle_{A}|H\rangle_{B}+$ $\sin \phi|V\rangle_{A}|V\rangle_{B}$, where the parameter $\phi$ is modulated by $\mathrm{H} 1$, a half-wave plate (HWP) put in front of the BBO crystal to adjust the polarization of the pump. Here the pump is a continuous-wave diode laser with $140 \mathrm{~mW}$ and the wavelength is $404 \mathrm{~nm}$. The fidelity between the experimental state and the theoretical state is beyond $98.80 \%$. The computational bases $|0\rangle$ and $|1\rangle$ are encoded in the horizontal polarization $|H\rangle$ and the vertical polarization $|V\rangle$ of the photons, respectively. The photon pair is separated spatially via a single mode fiber. One photon is sent to Alice and the other one is sent to Bob. In Alice part, as illustrated in Fig. 3, we use three beam displacers (BDs) in which the vertical-polarized photon remains on its path while the horizontal-polarized photon shifts down. The intraparticle entanglement is realized between the path and the polarization degrees of freedom of Alice's photon. The upper path state is encoded into $|0\rangle$, and the down path state corresponds to $|1\rangle$. After BD1, the photons in different polarization states travel two paths. Thus the polarization and the path are entangled. Due to the HWP at $45^{\circ}(\mathrm{H} 7)$, the horizontal and vertical polarizations exchange, whereafter the HWP in the upper path (H8) rotates the polarization. The angle $\theta$ modulated by $\mathrm{H} 8$ is the controllable parameter of the path-polarization coupling. Right after H8, there is another beam displacer, BD2, to fulfill the preparation of the intraparticle entanglement of Alice's photon. On the other hand, in Bob's part, the photons are measured directly. Finally we get the three-qubit states which contain intraparticle and external entanglement and can be described as

$$
|\psi\rangle=\cos \phi|1\rangle_{A_{1}}|1\rangle_{A_{2}}|0\rangle_{B}+\sin \phi|\varphi\rangle_{A_{1} A_{2}}|1\rangle_{B},
$$

where $|\varphi\rangle_{A_{1} A_{2}}=\cos \theta|0\rangle_{A_{1}}|1\rangle_{A_{2}}+\sin \theta|1\rangle_{A_{1}}|0\rangle_{A_{2}}$.

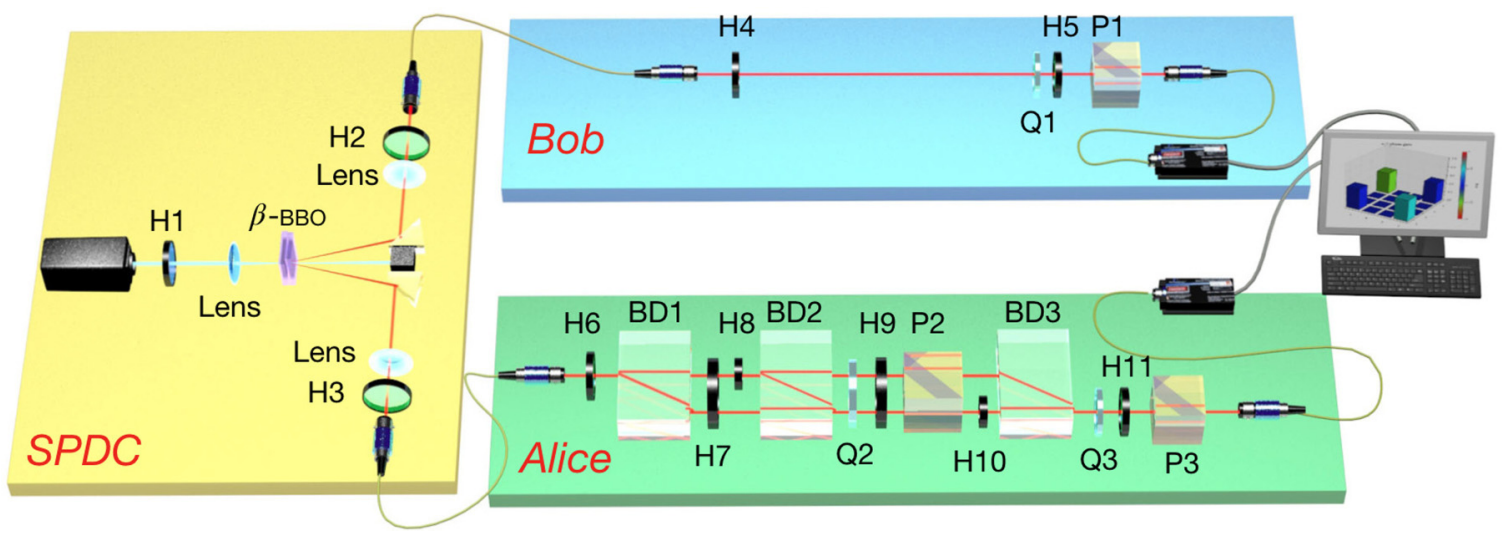

FIG. 3. Experimental setup. The polarization-entangled photon pairs are generated by the spontaneous parametric down-conversion process. In the Alice part, the polarization and path states are entangled. In each mode, half-wave plates, quarter-wave plates, and polarization beam splitters are set for state tomography. In the experiment, the photons are collected by two single-photon counting modules and identified by the coincidence counter. H, half-wave plate, H1-H11; Q, quarter-wave plate, Q1-Q3; P, polarization beam splitter, P1-P3; BD, beam displacer, BD1-BD3. 

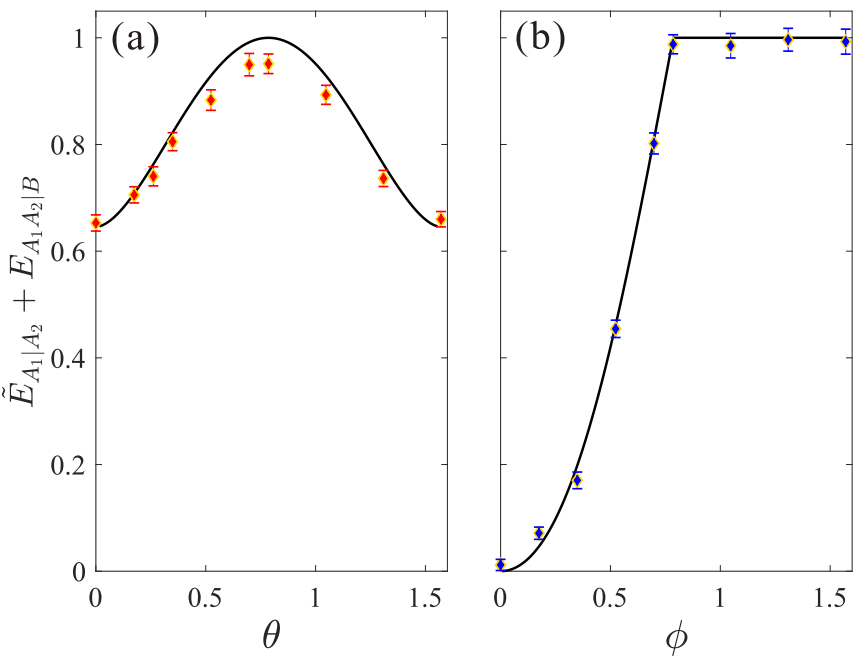

FIG. 4. $\tilde{E}_{A_{1} \mid A_{2}}+E_{A_{1} A_{2} \mid B}$ as a function of $\theta$ for the states (6) [panel (a)], and as a function of $\phi$ for the states (5) [panel (b)]. The dots are the experimental results and the curves are the theoretical predictions. The error bars are from the Poissonian distribution of photon counts and the uncertainty of the wave plates.

In order to reconstruct the density matrices of these threequbit states, we perform quantum state tomography for these states. According to the maximum likelihood estimation, the density matrices are reconstructed [58]. The project measurement of polarization is realized by a standard polarization tomography setup (SPTS), which consists of a quarter-wave plate (QWP), a half-wave plate, and a polarization beam splitter (PBS). As shown in Fig. 3, there are three such setups: (Q1,H5,P1), (Q2,H9,P2), and (Q3,H11,P3). The first two setups measure the polarization states of Bob and Alice, respectively. As for the last one, it is used to measure the path state of Alice. The following demonstrates how it works. Consider BD2, it is the last element in the state preparation. If a photon is in the upper path after BD2, its polarization is horizontal when it arrives at Q3, i.e., the last SPTS, and if it is in the down path, it will be vertical-polarized. Therefore, the last SPTS measures the path state via the polarization state tomography. The external entanglement is calculated from the density matrix of the complete system, i.e., the three-qubit state, and the intraparticle entanglement is calculated independently using reduced density matrix tomography in Alice's part and simple photon detection in Bob's part for coincidence counting. Besides it is necessary to mention that the HWP right after P2 in the down path $(\mathrm{H} 10)$ is at $45^{\circ}$.

We first fix the value of $\theta$ at $45^{\circ}$ and adjust $\mathrm{H} 1$ to change the values of $\phi$ from $0^{\circ}$ to $90^{\circ}$. Thus, the experimental state in Eq. (7) becomes the states in Eq. (5). If $\phi=90^{\circ}$, the intraparticle entanglement of system $A$ is maximal, and if $\phi=0^{\circ}$, the state is $|\psi\rangle=|110\rangle$, which is separable. Furthermore, the states with a fixed value of $\phi=45^{\circ}$ and adjustable values of $\theta$ from $0^{\circ}$ to $90^{\circ}$ are tested, which are the states in Eq. (6). We reconstruct the density matrices of all these states and then calculate their entanglements $\tilde{E}_{A_{1} \mid A_{2}}$ and $E_{A_{1} A_{2} \mid B}$ based on $E_{F}$ using the expressions given above. As shown in Fig. 4, the experimental results coincide with the theoretical predictions. Although the different values of $\theta$ and $\phi$ represent the different states, the sum of $\tilde{E}_{A_{1} \mid A_{2}}+E_{A_{1} A_{2} \mid B}$ will not exceed 1, which experimentally demonstrates the monogamy relation (3). We remark that $E_{A_{1} A_{2} \mid B}$ is evaluated using Eq. (4), which is valid only for genuine pure states. However, the actual entanglement $E_{A_{1} A_{2} \mid B}$ of the experimental state, which is not exactly pure, is lower than the value obtained from Eq. (4) $[48,50]$. On the other hand, we choose $\tilde{E}_{A_{1} \mid A_{2}}$ as the horizontal ordinate and $E_{A_{1} A_{2} \mid B}$ as the vertical ordinate to plot Fig. 2. We find that for the states (5) $E_{A_{1} A_{2} \mid B}$ first increases and then decreases as $\tilde{E}_{A_{1} \mid A_{2}}$ increases; but for the states (6) $E_{A_{1} A_{2} \mid B}$ is almost invariant. All the experimental results agree with the theoretical results. Moreover, these values are in the area below the straight line $E_{A_{1} A_{2} \mid B}=1-\tilde{E}_{A_{1} \mid A_{2}}$.

In this experiment, the visibility of the Mach-Zehnder interferometer is about 100:1 and the average fidelity [59] between the experimental states and theoretical states is $98.86 \pm 0.41 \%$. Moreover, the negativity in inequality (8) has been evaluated for the mixed experimental states. Besides, the single-photon avalanche photon-diode (SPCM-3369) is used to detect photons whose detection efficiency is $68 \%$. The detection events from the same pair are identified by a coincidence counter as long as they arrive within \pm 3 ns. In addition, the coincidence counts are about $1000 \mathrm{~s}^{-1}$ and we record clicks for $10 \mathrm{~s}$. There are many sources of the measurement uncertainty, such as counting statistics, detector efficiency, the detector's dead time, timing uncertainty, and the alignment error of wave plates. However, the resulting uncertainty is dominated by counting statistics [60], which we have calculated via the Poissonian distribution and the errors introduced by wave plates cannot be ignored. Both of them are shown in the figure.

\section{OTHER MONOGAMY INEQUALITIES}

Now we show that the tradeoff between intraparticle and external entanglement is not restricted to some specific measures of entanglement, and it can be shown by other entanglement measures as well, such as the negativity and concurrence. In Ref. [48], Camalet also presented a monogamy inequality involving only one entanglement monotone, the negativity $E_{N}$. For the bipartite state $\varrho_{A B}$, the negativity is defined by $E_{N}\left(\varrho_{A B}\right)=\left(\left\|\varrho_{A B}^{T_{B}}\right\|-1\right) / 2[54,55]$, where $\|\cdot\|$ is the trace norm and $T_{B}$ is the partial transpose with respect to system $B$. Contrary to other entanglement measures, such as $E_{F}$ or $E_{F}^{\prime}, E_{N}$ is readily computable for any state. For the three-qubit state $\varrho_{A_{1} A_{2} B}$, the monogamy inequality is

$$
E_{N}\left(\varrho_{A_{1} A_{2}}\right)+g\left[E_{N}\left(\varrho_{A_{1} A_{2} \mid B}\right)\right] \leqslant E_{N, \max },
$$

where $E_{N \text {, max }}$, the maximum value of $E_{N}$, is equal to $1 / 2$ for the two-qubit states, and the nondecreasing function $g$ is given by $g(x)=\left(3 / 2-\sqrt{1-2 x^{2}}-\sqrt{1 / 4-x^{2}}\right) / 2$ [48]. The monogamy inequality (8) has been calculated for our experimentally realized states in Appendix B.

Many familiar entanglement monotones satisfy monogamy inequalities of the form of Eq. (8) [51], but determining explicitly the corresponding function $g$ may not be always possible. Now we present another case for which this can 
be achieved. For the qubit-qudit pure state $\left|\phi_{A B}\right\rangle$, the concurrence is defined by $C\left(\left|\phi_{A B}\right\rangle\right)=\sqrt{2\left(1-\operatorname{Tr} \varrho_{B}^{2}\right)}[3,4]$, where $\varrho_{B}=\operatorname{Tr}_{A}\left(\left|\phi_{A B}\right\rangle\left\langle\phi_{A B}\right|\right)$ is the reduced density operator of system $B$. It is generalized to mixed states via the convex roof extension $[3,4]$. For the three-qubit state $\varrho_{A_{1} A_{2} B}$, the following monogamy inequality holds:

$$
C\left(\varrho_{A_{1} A_{2}}\right)+\tilde{g}\left[C\left(\varrho_{A_{1} A_{2} \mid B}\right)\right] \leqslant C_{\max },
$$

where $C_{\max }$, the maximum value of $C$, is equal to 1 for the two-qubit states, and the nondecreasing function $\tilde{g}$ is given by $\tilde{g}(x)=\left(1-\sqrt{1-x^{2}}\right) / 2$. The monogamy inequality (9) has also been calculated for our experimentally realized states in Appendix C.

\section{CONCLUSION}

We have experimentally observed the tradeoff relation between intraparticle and external entanglement in a photonic system. Although the experimental states are not exactly pure states, the monogamy inequality (3) still holds for experimental mixed states. This realization has verified the theoretical prediction that the entanglement between different degrees of freedom of a quantum single particle can restrict its entanglement with other particles. This property may have applications in quantum information, such as the construction of quantum communication networks. Our experiment opens the door to experimentally investigating the distribution of different kinds of entanglement in multipartite systems and points a way to generate external entanglement by decreasing internal entanglement. Furthermore, our experiment will be greatly helpful for further research on the other monogamy relations in multipartite systems. For instance, one can demonstrate the inequality between local coherence and entanglement [48,61] and the inequality between intraparticle entanglement and external correlations [51].

\section{ACKNOWLEDGMENTS}

This work is funded by the National Natural Science Foundation of China (Grants No. 11504253, No. 11575051, No. 11674306, No. 61590932, and No. 11734015), the National Key R\&D Program (Grants No. 2016 YFA0301300 and No. 2016A0301700), the Anhui Initiative in Quantum Information Technologies, the K.C. Wong Magna Fund in Ningbo University, and the Hebei NSF (Grant No. A2016205215).

\section{APPENDIX A: INEQUALITY (2) FOR THE NEGATIVITY $\boldsymbol{E}_{\boldsymbol{N}}$}

If we use the negativity $E_{N}$ to quantify the internal entanglement between $A_{1}$ and $A_{2}$, the inequality (2) in the main text becomes

$$
E_{N}\left(\varrho_{A_{1} A_{2}}\right)+E_{N}^{\prime}\left(|\psi\rangle_{A_{1} A_{2} \mid B}\right) \leqslant \frac{1}{2}
$$

where $E_{N}\left(\varrho_{A_{1} A_{2}}\right)=\left(\left\|\varrho_{A_{1} A_{2}}^{T_{A_{2}}}\right\|-1\right) / 2$, and $E_{N \text {, max }}$, the maximum value of $E_{N}\left(\varrho_{A_{1} A_{2}}\right)$, is equal to $1 / 2$ for the two-qubit state $\varrho_{A_{1} A_{2}}$. The external entanglement $E_{N}^{\prime}\left(|\psi\rangle_{A_{1} A_{2} \mid B}\right)$ is

$$
E_{N}^{\prime}\left(|\psi\rangle_{A_{1} A_{2} \mid B}\right)=\frac{1}{2}-E_{N}\left(\varrho_{A_{1} A_{2}}^{\prime}\right)
$$

with

$$
\begin{aligned}
E_{N}\left(\varrho_{A_{1} A_{2}}^{\prime}\right)= & \max _{U} E_{N}\left(U \varrho_{A_{1} A_{2}} U^{\dagger}\right) \\
= & \max \left\{0, \frac{1}{2} \sqrt{\left(\lambda_{1}-\lambda_{3}\right)^{2}+\left(\lambda_{2}-\lambda_{4}\right)^{2}}\right. \\
& \left.-\frac{\lambda_{2}}{2}-\frac{\lambda_{4}}{2}\right\},
\end{aligned}
$$

where $U$ denotes the unitary operators of $A, \varrho_{A_{1} A_{2}}^{\prime}$ is the density operator corresponding to the maximum over $U$, and $\left\{\lambda_{i}\right\}$ are the eigenvalues of $\varrho_{A_{1} A_{2}}$ in nonascending order [53].

Now we consider a class of three-qubit pure states with one parameter $\phi$,

$$
|\Psi\rangle=\cos \phi|110\rangle+\sin \phi \frac{|01\rangle+|10\rangle}{\sqrt{2}}|1\rangle .
$$

Based on Eqs. (A1)-(A3), one can obtain its internal and external entanglement measured by the negativity:

$$
\begin{aligned}
& E_{N}\left(\varrho_{A_{1} A_{2}}\right)=\frac{1}{4} \sqrt{3+\cos (4 \phi)}-\frac{1}{2} \cos ^{2} \phi, \\
& E_{N}^{\prime}\left(|\psi\rangle_{A_{1} A_{2} \mid B}\right)=\frac{1}{2}+\frac{1}{2} \min \left\{\cos ^{2} \phi, \sin ^{2} \phi\right\} \\
& -\frac{1}{4} \sqrt{3+\cos (4 \phi)} \text {. }
\end{aligned}
$$

Moreover, we also consider the pure state with parameter $\theta$,

$$
|\Phi\rangle=\frac{1}{\sqrt{2}}|110\rangle+\frac{\cos \theta|01\rangle+\sin \theta|10\rangle}{\sqrt{2}}|1\rangle .
$$

Based on Eqs. (A1)-(A3), one can obtain its internal and external entanglement measured by the negativity:

$$
\begin{gathered}
E_{N}\left(\varrho_{A_{1} A_{2}}\right)=\frac{1}{8}(-2+\sqrt{6-2 \cos (4 \theta)}), \\
E_{N}^{\prime}\left(|\psi\rangle_{A_{1} A_{2} \mid B}\right)=\frac{1}{4}(3-\sqrt{2}) .
\end{gathered}
$$

The theoretical and experimental results have been shown in Fig. 5. We can see that all the sums of internal and external entanglement are bounded by $1 / 2$; i.e., the inequality (A1) always holds.

\section{APPENDIX B: THE MONOGAMY INEQUALITY (8) INVOLVING ONLY $E_{N}$}

In Ref. [48], the author also presented a monogamy inequality involving only one entanglement monotone, the negativity $E_{N}$. For the bipartite state $\varrho_{A B}$, the negativity is defined by $E_{N}\left(\varrho_{A B}\right)=\left(\left\|\varrho_{A B}^{T_{B}}\right\|-1\right) / 2$, where $\|\cdot\|$ is the trace norm and $T_{B}$ is the partial transpose with respect to system $B$. For the three-qubit pure state $|\psi\rangle_{A_{1} A_{2} B}$, the monogamy inequality is

$$
E_{N}\left(\varrho_{A_{1} A_{2}}\right)+g\left[E_{N}\left(|\psi\rangle_{A_{1} A_{2} \mid B}\right)\right] \leqslant \frac{1}{2},
$$

where $E_{N \text {, max }}$, the maximum value of $E_{N}\left(\varrho_{A_{1} A_{2}}\right)$, is equal to $1 / 2$ for the two-qubit state $\varrho_{A_{1} A_{2}}$, and the nondecreasing function $g$ is given by

$$
g(x)=\frac{3}{4}-\frac{\sqrt{1-2 x^{2}}}{2}-\frac{\sqrt{1-4 x^{2}}}{4},
$$

when the number of nonzero eigenvalues of $\varrho_{A_{1} A_{2}}$ is equal to 2 [48]. 

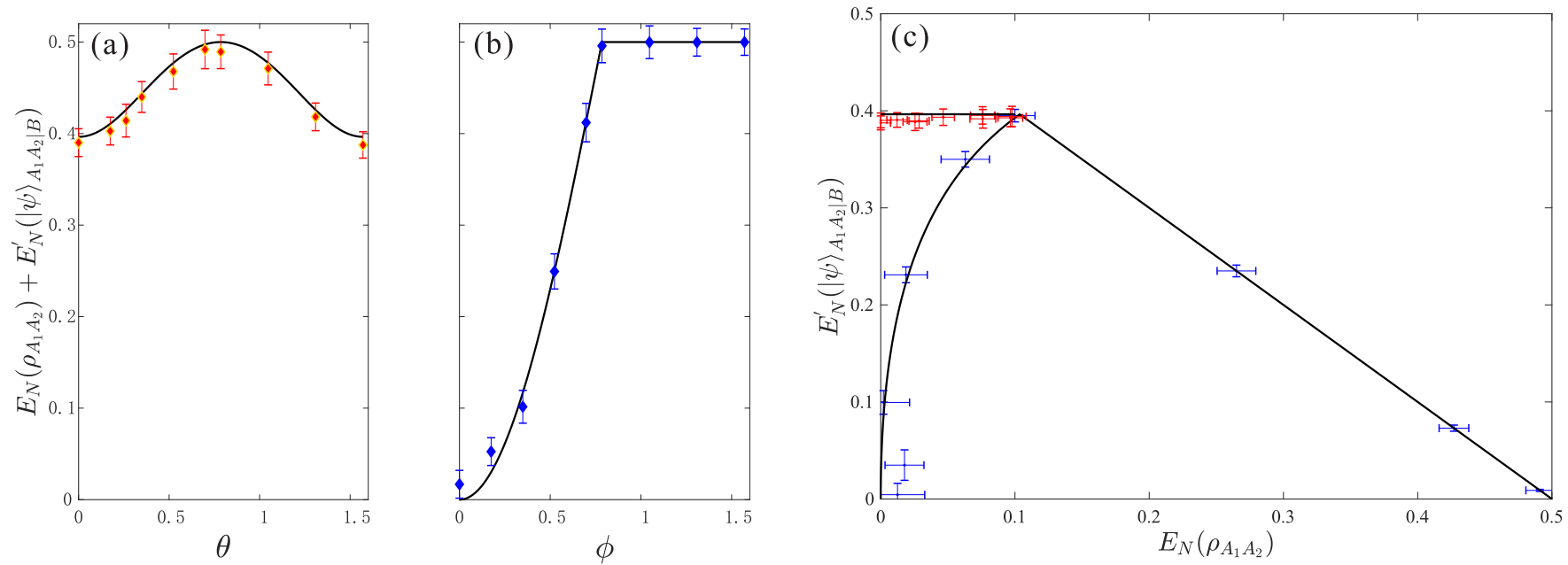

FIG. 5. We use the negativities $E_{N}\left(\varrho_{A_{1} A_{2}}\right)$ and $E_{N}^{\prime}\left(|\psi\rangle_{A_{1} A_{2} \mid B}\right)$ to quantify the entanglement among these three qubits. The red and blue dots are experimental results and the lines are theoretical predictions.

Now we consider a class of three-qubit pure states with one parameter $\phi$,

$$
|\Psi\rangle=\cos \phi|110\rangle+\sin \phi \frac{|01\rangle+|10\rangle}{\sqrt{2}}|1\rangle .
$$

Based on Eqs. (B1) and (B2), one can obtain its internal and external entanglement measured by the negativity:

$$
\begin{aligned}
& E_{N}\left(\varrho_{A_{1} A_{2}}\right)=\frac{1}{4} \sqrt{3+\cos (4 \phi)}-\frac{1}{2} \cos ^{2} \phi \\
& g\left[E_{N}\left(|\psi\rangle_{A_{1} A_{2} \mid B}\right)\right]= \frac{3}{4}-\frac{\sqrt{1-2 \cos ^{2} \phi \sin ^{2} \phi}}{2} \\
&-\frac{\sqrt{1-4 \cos ^{2} \phi \sin ^{2} \phi}}{4} .
\end{aligned}
$$

Moreover, we also consider the pure state with parameter $\theta$,

$$
|\Phi\rangle=\frac{1}{\sqrt{2}}|110\rangle+\frac{\cos \theta|01\rangle+\sin \theta|10\rangle}{\sqrt{2}}|1\rangle .
$$

Based on Eqs. (B1) and (B2), one can obtain its internal and external entanglement measured by the negativity:

$$
\begin{gathered}
E_{N}\left(\varrho_{A_{1} A_{2}}\right)=\frac{1}{8}(-2+\sqrt{6-2 \cos (4 \theta)}), \\
g\left[E_{N}\left(|\psi\rangle_{A_{1} A_{2} \mid B}\right)\right]=\frac{1}{4}(3-\sqrt{2}) .
\end{gathered}
$$

The theoretical and experimental results have been shown in Fig. 6. We can see that all the sums of internal and external entanglement are bounded by $1 / 2$; i.e., the inequality (B1) always holds.

\section{APPENDIX C: THE MONOGAMY INEQUALITY (9) INVOLVING ONLY $C$}

Proposition 1. For the three-qubit state $\varrho_{A_{1} A_{2} B}$, the internal entanglement $C\left(\varrho_{A_{1} A_{2}}\right)$ and the external entanglement $C\left(\varrho_{A_{1} A_{2} \mid B}\right)$, as quantified by the concurrence, obey the monogamy relation

$$
C\left(\varrho_{A_{1} A_{2}}\right)+\tilde{g}\left[C\left(\varrho_{A_{1} A_{2} \mid B}\right)\right] \leqslant 1,
$$

where 1 is the maximal value of $C$ for two-qubit states and

$$
\tilde{g}(x)=\frac{1-\sqrt{1-x^{2}}}{2} .
$$

Proof. For a pure three-qubit state, the external entanglement is given by

$$
C\left(|\psi\rangle_{A_{1} A_{2} \mid B}\right)=\sqrt{2\left(1-\operatorname{Tr} \varrho_{A_{1} A_{2}}^{2}\right)}=2 \sqrt{\lambda_{1}\left(1-\lambda_{1}\right)},
$$

with $\lambda_{1}$ being the maximal eigenvalue of $\varrho_{A_{1} A_{2}}$. Since the above function of $\varrho_{A_{1} A_{2}}$ is concave and the concurrence is defined via the convex roof extension for mixed states, the external entanglement obeys

$$
C\left(\varrho_{A_{1} A_{2} \mid B}\right) \leqslant\left\langle 2 \sqrt{\Lambda_{1}\left(1-\Lambda_{1}\right)}\right\rangle,
$$

for a general three-qubit state $\varrho_{A_{1} A_{2} B}=\sum_{i} p_{i}\left|\psi_{i}\right\rangle\left\langle\psi_{i}\right|$ with $\left\langle f\left(\Lambda_{1}\right)\right\rangle=\sum_{i} p_{i} f\left(\lambda_{1, i}\right)$ and $\lambda_{1, i}$ being the maximal eigenvalue of $\operatorname{Tr}_{B}\left|\psi_{i}\right\rangle\left\langle\psi_{i}\right|$. The internal entanglement can be obtained by the formula $C\left(\varrho_{A_{1} A_{2}}\right)=\max \left\{0, \sigma_{1}-\sigma_{2}-\sigma_{3}-\right.$ $\left.\sigma_{4}\right\}$, with $\left\{\sigma_{i}\right\}$ being the square roots of eigenvalues of $\varrho_{A_{1} A_{2}} \sigma_{y} \otimes \sigma_{y} \varrho_{A_{1} A_{2}}^{*} \sigma_{y} \otimes \sigma_{y}$ in decreasing order [4]. In the bipartite partition $A_{1} A_{2} \mid B$, the internal entanglement can be maximized via two-qubit unitary transformations on $A_{1} A_{2}$, and the following relation holds:

$$
C\left(\varrho_{A_{1} A_{2}}\right) \leqslant \max _{U} C\left(U \varrho_{A_{1} A_{2}} U^{\dagger}\right)
$$

where the equality is satisfied for the so-called maximally entangled mixed state (MEMS) $\varrho_{A_{1} A_{2}}^{\prime}$ [53]. In the case of two-qubit MEMSs [53], its concurrence is $C\left(\varrho_{A_{1} A_{2}}^{\prime}\right)=$ $\max \left\{0, \lambda_{1}-\lambda_{3}-2 \sqrt{\lambda_{2} \lambda_{4}}\right\} \leqslant \lambda_{1}$, and hence $C\left(\varrho_{A_{1} A_{2}}\right) \leqslant$ $\lambda_{1} \leqslant\left\langle\Lambda_{1}\right\rangle$. Therefore, we have

$$
\begin{aligned}
C\left(\varrho_{A_{1} A_{2}}\right)+\tilde{g}\left[C\left(\varrho_{A_{1} A_{2} \mid B}\right)\right] & \leqslant\left\langle\Lambda_{1}+\tilde{g}\left(2 \sqrt{\Lambda_{1}\left(1-\Lambda_{1}\right)}\right)\right\rangle \\
& =\left\langle\Lambda_{1}+\frac{1-\sqrt{1-4 \Lambda_{1}\left(1-\Lambda_{1}\right)}}{2}\right\rangle \\
& =\left\langle\Lambda_{1}+\frac{1-\left(2 \Lambda_{1}-1\right)}{2}\right\rangle \\
& =1,
\end{aligned}
$$



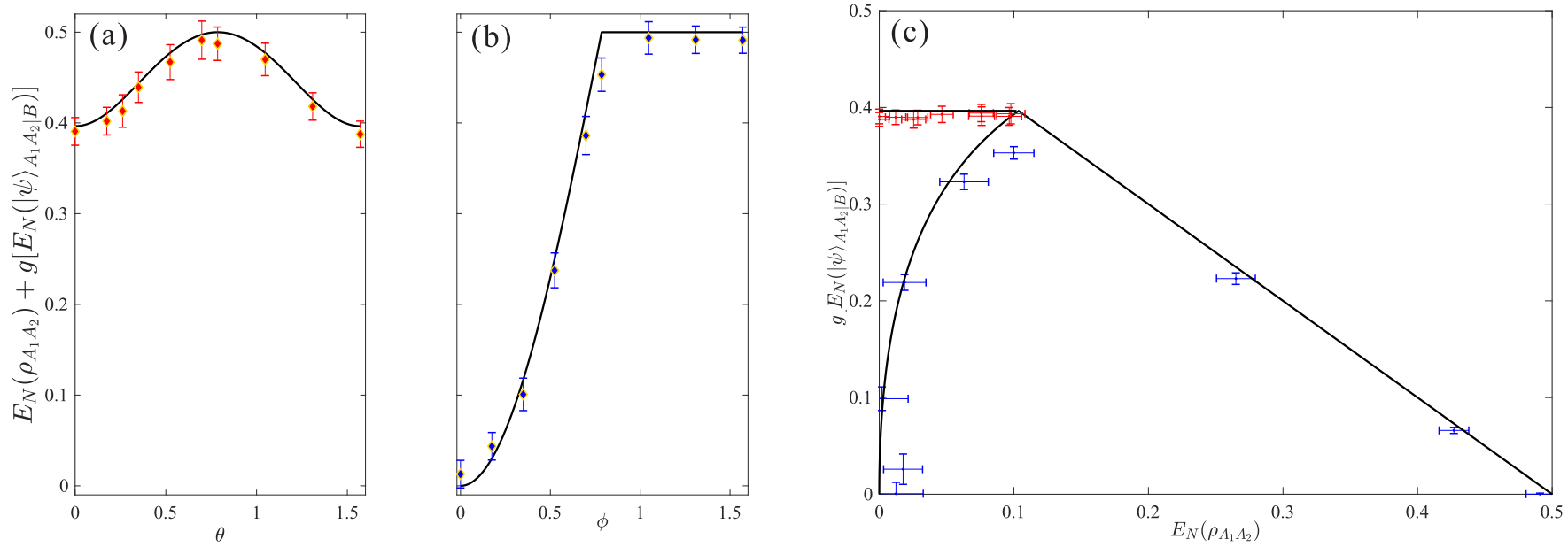

FIG. 6. In order to use the same measure, $E_{N}\left(\varrho_{A_{1} A_{2}}\right)$ and $E_{N}\left(|\psi\rangle_{A_{1} A_{2} \mid B}\right)$, to quantify the entanglement, we employ the function $g$. Note that $g$ is not shown in the figure. The red and blue dots are experimental results and the lines are theoretical predictions.

where $1 / 2 \leqslant \lambda_{1, i} \leqslant 1$ and the monotonicity and convexity of $\tilde{g}$ have been used. Thus, we obtain the monogamy relation (C1).

Now we consider a class of three-qubit pure states with one parameter $\phi$,

$$
|\Psi\rangle=\cos \phi|110\rangle+\sin \phi \frac{|01\rangle+|10\rangle}{\sqrt{2}}|1\rangle .
$$

One can obtain its internal and external entanglement measured by concurrence:

$$
\begin{gathered}
C\left(\varrho_{A_{1} A_{2}}\right)=\sin ^{2} \phi, \\
\tilde{g}\left[C\left(|\psi\rangle_{A_{1} A_{2} \mid B}\right)\right]=1-\lambda_{1},
\end{gathered}
$$

with

$$
\lambda_{1}=\max \left\{\cos ^{2} \phi, \sin ^{2} \phi\right\} .
$$

One can see that if $\lambda_{1}=\sin ^{2} \phi$ (i.e., $\sin ^{2} \phi \geqslant \cos ^{2} \phi$ ), then $C\left(\varrho_{A_{1} A_{2}}\right)+\tilde{g}\left[C\left(|\psi\rangle_{A_{1} A_{2} \mid B}\right)\right]=1$ holds. Moreover, we also
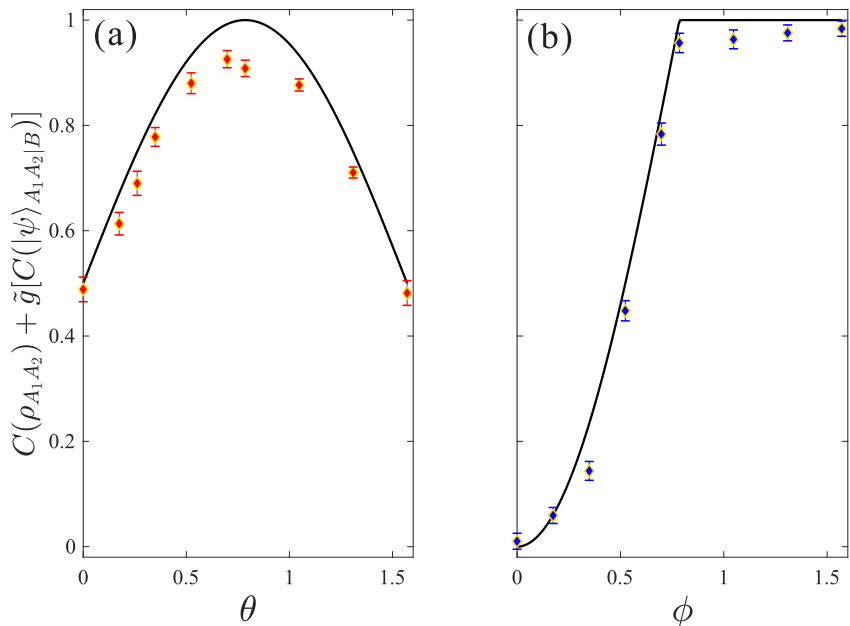

consider the pure state with parameter $\theta$,

$$
|\Phi\rangle=\frac{1}{\sqrt{2}}|110\rangle+\frac{\cos \theta|01\rangle+\sin \theta|10\rangle}{\sqrt{2}}|1\rangle .
$$

One can obtain its internal and external entanglement measured by concurrence:

$$
\begin{gathered}
C\left(\varrho_{A_{1} A_{2}}\right)=\frac{1}{2}|\sin (2 \theta)|, \\
\tilde{g}\left[C\left(|\psi\rangle_{A_{1} A_{2} \mid B}\right)\right]=1-\lambda_{1}=\frac{1}{2},
\end{gathered}
$$

with $\lambda_{1}=1 / 2$.

The theoretical and experimental results have been shown in Fig. 7. We can see that all the sums of internal and external entanglement are bounded by 1 ; i.e., the inequality $(\mathrm{C} 1)$ always holds.

\section{APPENDIX D: QUANTUM STATE TOMOGRAPHY}

We performed tomography on all the states we prepared. Their density matrices are described in Eq. (7) of the main text. First, $\theta$ is fixed at $45^{\circ}$, but $\phi$ is changing. Then we change

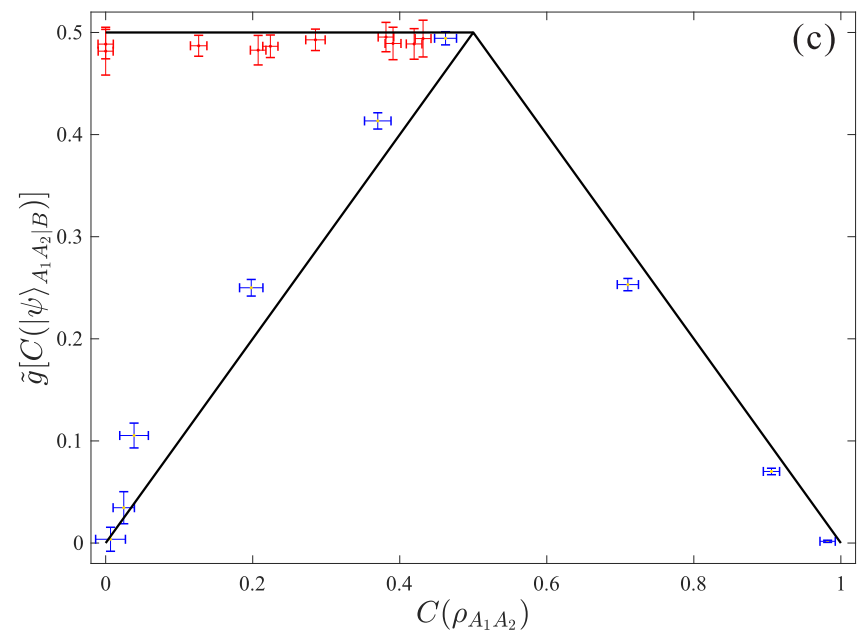

FIG. 7. We use the concurrence $C$ to quantify the internal and external entanglement with the function $\tilde{g}$. The red and blue dots are experimental results and the lines are theoretical predictions. 
TABLE I. Fidelities for the quantum state Eq. (7) of the main text. (i) We change the value of $\theta$ with the fixed $\phi$ at $45^{\circ}$; (ii) $\theta$ is fixed at $45^{\circ}$ but $\phi$ is changing from $0^{\circ}$ to $90^{\circ}$. The average fidelity of all these states is $98.86 \pm 0.41 \%$.

\begin{tabular}{|c|c|c|c|c|c|}
\hline$\theta$ & $0^{\circ}$ & $10^{\circ}$ & $15^{\circ}$ & $20^{\circ}$ & $30^{\circ}$ \\
\hline Fidelity & $98.17 \pm 0.13 \%$ & $99.17 \pm 0.12 \%$ & $98.77 \pm 0.02 \%$ & $99.28 \pm 0.03 \%$ & $98.39 \pm 0.11 \%$ \\
\hline$\theta$ & $40^{\circ}$ & $45^{\circ}$ & $60^{\circ}$ & $75^{\circ}$ & $90^{\circ}$ \\
\hline Fidelity & $98.85 \pm 0.03 \%$ & $98.15 \pm 0.01 \%$ & $98.52 \pm 0.04 \%$ & $98.72 \pm 0.08 \%$ & $98.96 \pm 0.09 \%$ \\
\hline$\phi$ & $0^{\circ}$ & $15^{\circ}$ & $30^{\circ}$ & $45^{\circ}$ & $50^{\circ}$ \\
\hline Fidelity & $99.05 \pm 0.11 \%$ & $99.16 \pm 0.07 \%$ & $99.51 \pm 0.02 \%$ & $99.16 \pm 0.05 \%$ & $98.94 \pm 0.05 \%$ \\
\hline$\phi$ & $60^{\circ}$ & $70^{\circ}$ & $80^{\circ}$ & $90^{\circ}$ & Average \\
\hline Fidelity & $99.10 \pm 0.06 \%$ & $98.77 \pm 0.03 \%$ & $98.20 \pm 0.12 \%$ & $99.39 \pm 0.05 \%$ & $98.86 \pm 0.41 \%$ \\
\hline
\end{tabular}

the value of $\theta$ with the fixed $\phi$ at $45^{\circ}$. We can get their density matrices that are obtained by maximum likelihood estimation. In Table I, we list the fidelity of all the states which only change the value of $\phi$ or $\theta$.

Although the experimental states are not exactly pure states, the monogamy inequality (3) in the main text still holds for experimental mixed states. Suppose that the experimentally realized tripartite state is $\varrho_{A_{1} A_{2} B}$, thus the external entanglement is defined by the convex roof,

$$
E_{F}^{\prime}\left(\varrho_{A_{1} A_{2} \mid B}\right)=\inf _{\left\{p_{i},\left|\psi_{i}\right\rangle_{A_{1} A_{2} B}\right\}} \sum_{i} p_{i} E_{F}^{\prime}\left(\left|\psi_{i}\right\rangle_{A_{1} A_{2} \mid B}\right) .
$$

We assume that $\varrho_{A_{1} A_{2} B}=\sum_{j} p_{j}\left|\psi_{j}\right\rangle\left\langle\psi_{j}\right|$ is the optimal decomposition for $\varrho_{A_{1} A_{2} B}$ to achieve the above infimum. Therefore,

$$
\begin{aligned}
& E_{F}\left(\varrho_{A_{1} A_{2}}\right)+E_{F}^{\prime}\left(\varrho_{A_{1} A_{2} \mid B}\right) \\
& \quad=E_{F}\left(\varrho_{A_{1} A_{2}}\right)+\sum_{j} p_{j} E_{F}^{\prime}\left(\left|\psi_{j}\right\rangle_{A_{1} A_{2} \mid B}\right) \\
& \quad=E_{F}\left(\varrho_{A_{1} A_{2}}\right)+1-\sum_{j} p_{j} E_{F}\left(U_{j} \varrho_{j, A_{1} A_{2}} U_{j}^{\dagger}\right)
\end{aligned}
$$

$$
\begin{aligned}
& \leqslant E_{F}\left(\varrho_{A_{1} A_{2}}\right)+1-\sum_{j} p_{j} E_{F}\left(V \varrho_{j, A_{1} A_{2}} V^{\dagger}\right) \\
& \leqslant E_{F}\left(\varrho_{A_{1} A_{2}}\right)+1-E_{F}\left(V \sum_{j} p_{j} \varrho_{j, A_{1} A_{2}} V^{\dagger}\right) \\
& =E_{F}\left(\varrho_{A_{1} A_{2}}\right)+1-E_{F}\left(\varrho_{A_{1} A_{2}}^{\prime}\right) \\
& \leqslant 1
\end{aligned}
$$

where $\quad \varrho_{j, A_{1} A_{2}}=\operatorname{Tr}_{B}\left|\psi_{j}\right\rangle\left\langle\psi_{j}\right|, \quad E_{F}^{\prime}\left(\left|\psi_{j}\right\rangle_{A_{1} A_{2} \mid B}\right)=1-$ $E_{F}\left(\varrho_{j, A_{1} A_{2}}^{\prime}\right), \quad E_{F}\left(\varrho_{j, A_{1} A_{2}}^{\prime}\right)=\max _{U} E_{F}\left(U \varrho_{j, A_{1} A_{2}} U^{\dagger}\right)=$ $E_{F}\left(U_{j} \varrho_{j, A_{1} A_{2}} U_{j}^{\dagger}\right), \quad E_{F}\left(\varrho_{A_{1} A_{2}}^{\prime}\right)=\max _{U} E_{F}\left(U \varrho_{A_{1} A_{2}} U^{\dagger}\right)=$ $E_{F}\left(V \varrho_{A_{1} A_{2}} V^{\dagger}\right)$, the first inequality holds since $E_{F}\left(U_{j} \varrho_{j, A_{1} A_{2}} U_{j}^{\dagger}\right)=\max _{U} E_{F}\left(U \varrho_{j, A_{1} A_{2}} U^{\dagger}\right) \geqslant E_{F}\left(V \varrho_{j, A_{1} A_{2}} V^{\dagger}\right)$, and the second inequality is from the convex property of $E_{F}$. This result also follows from the fact that $E_{F}^{\prime}$ is a concave function of $\varrho_{A 1 A 2}$, as shown by Proposition 2 of the Supplemental Material of Ref. [48].
[1] R. Horodecki, P. Horodecki, M. Horodecki, and K. Horodecki, Quantum entanglement, Rev. Mod. Phys. 81, 865 (2009).

[2] C. H. Bennett, H. J. Bernstein, S. Popescu, and B. Schumacher, Concentrating partial entanglement by local operations, Phys. Rev. A 53, 2046 (1996).

[3] V. Coffman, J. Kundu, and W. K. Wootters, Distributed entanglement, Phys. Rev. A 61, 052306 (2000).

[4] W. K. Wootters, Entanglement of Formation of an Arbitrary State of Two Qubits, Phys. Rev. Lett. 80, 2245 (1998).

[5] T. J. Osborne and F. Verstraete, General Monogamy Inequality for Bipartite Qubit Entanglement, Phys. Rev. Lett. 96, 220503 (2006).

[6] G. Adesso and F. Illuminati, Continuous variable tangle, monogamy inequality, and entanglement sharing in Gaussian states of continuous variable systems, New J. Phys. 8, 15 (2006).

[7] T. Hiroshima, G. Adesso, and F. Illuminati, Monogamy Inequality for Distributed Gaussian Entanglement, Phys. Rev. Lett. 98, 050503 (2007).

[8] G. Adesso and F. Illuminati, Strong Monogamy of Bipartite and Genuine Multipartite Entanglement: The Gaussian Case, Phys. Rev. Lett. 99, 150501 (2007).
[9] G. Adesso and F. Illuminati, Genuine multipartite entanglement of symmetric Gaussian states: Strong monogamy, unitary localization, scaling behavior, and molecular sharing structure, Phys. Rev. A 78, 042310 (2008).

[10] M. Christandl and A. Winter, "Squashed entanglement": An additive entanglement measure, J. Math. Phys. (NY) 45, 829 (2004).

[11] M. Koashi and A. Winter, Monogamy of quantum entanglement and other correlations, Phys. Rev. A 69, 022309 (2004).

[12] Y.-C. Ou and H. Fan, Monogamy inequality in terms of negativity for three-qubit states, Phys. Rev. A 75, 062308 (2007).

[13] J. S. Kim, A. Das, and B. C. Sanders, Entanglement monogamy of multipartite higher-dimensional quantum systems using convex-roof extended negativity, Phys. Rev. A 79, 012329 (2009).

[14] J. S. Kim, Negativity and tight constraints of multiqubit entanglement, Phys. Rev. A 97, 012334 (2018).

[15] G. W. Allen and D. A. Meyer, Polynomial Monogamy Relations for Entanglement Negativity, Phys. Rev. Lett. 118, 080402 (2017). 
[16] Y.-K. Bai, Y.-F. Xu, and Z. D. Wang, General Monogamy Relation for the Entanglement of Formation in Multiqubit Systems, Phys. Rev. Lett. 113, 100503 (2014).

[17] Y.-K. Bai, Y.-F. Xu, and Z. D. Wang, Hierarchical monogamy relations for the squared entanglement of formation in multipartite systems, Phys. Rev. A 90, 062343 (2014).

[18] T. R. de Oliveira, M. F. Cornelio, and F. F. Fanchini, Monogamy of entanglement of formation, Phys. Rev. A 89, 034303 (2014).

[19] Y.-K. Bai, M.-Y. Ye, and Z. D. Wang, Entanglement monogamy and entanglement evolution in multipartite systems, Phys. Rev. A 80, 044301 (2009).

[20] J. S. Kim and B. C. Sanders, Monogamy of multi-qubit entanglement using Rényi entropy, J. Phys. A: Math. Theor. 43, 445305 (2010).

[21] M. F. Cornelio and M. C. de Oliveira, Strong superadditivity and monogamy of the Rényi measure of entanglement, Phys. Rev. A 81, 032332 (2010).

[22] J. H. Choi and J. S. Kim, Negativity and strong monogamy of multiparty quantum entanglement beyond qubits, Phys. Rev. A 92, 042307 (2015).

[23] W. Song, Y.-K. Bai, M. Yang, M. Yang, and Z.-L. Cao, General monogamy relation of multiqubit systems in terms of squared Rényi- $\alpha$ entanglement, Phys. Rev. A 93, 022306 (2016).

[24] G.-M. Yuan, W. Song, M. Yang, D.-C. Li, J.-L. Zhao, and Z.-L. Cao, Monogamy relation of multi-qubit systems for squared Tsallis- $q$ entanglement, Sci. Rep. 6, 28719 (2016).

[25] G. Gour, S. Bandyopadhyay, and B. C. Sanders, Dual monogamy inequality for entanglement, J. Math. Phys. 48, 012108 (2007).

[26] C.-S. Yu and H.-S. Song, Entanglement monogamy of tripartite quantum states, Phys. Rev. A 77, 032329 (2008).

[27] R. Prabhu, A. K. Pati, A. Sen(De), and U. Sen, Relating monogamy of quantum correlations and multisite entanglement, Phys. Rev. A 86, 052337 (2012).

[28] R. Prabhu, A. K. Pati, A. Sen(De), and U. Sen, Conditions for monogamy of quantum correlations: Greenberger-HorneZeilinger versus $W$ states, Phys. Rev. A 85, 040102(R) (2012).

[29] X.-N. Zhu and S.-M. Fei, Entanglement monogamy relations of qubit systems, Phys. Rev. A 90, 024304 (2014).

[30] X.-N. Zhu and S.-M. Fei, Generalized monogamy relations of concurrence for $N$-qubit systems, Phys. Rev. A 92, 062345 (2015).

[31] X.-N. Zhu, X. Li-Jost, and S.-M. Fei, Monogamy relations of concurrence for any dimensional quantum systems, Quantum Inf. Process. 16, 279 (2017)

[32] Y.-C. Ou, H. Fan, and S.-M. Fei, Proper monogamy inequality for arbitrary pure quantum states, Phys. Rev. A 78, 012311 (2008).

[33] B. Regula, S. D. Martino, S. Lee, and G. Adesso, Strong Monogamy Conjecture for Multiqubit Entanglement: The FourQubit Case, Phys. Rev. Lett. 113, 110501 (2014).

[34] B. Regula, A. Osterloh, and G. Adesso, Strong monogamy inequalities for four qubits, Phys. Rev. A 93, 052338 (2016).

[35] A. Osterloh, Three-tangle of the nine classes of four-qubit states, Phys. Rev. A 94, 012323 (2016).
[36] Y. Luo and Y. Li, Monogamy of $\alpha$ th power entanglement measurement in qubit systems, Ann. Phys. (NY) 362, 511 (2015).

[37] Y. Luo, T. Tian, L.-H. Shao, and Y. Li, General Monogamy of Tsallis $q$-Entropy Entanglement in Multiqubit Systems, Phys. Rev. A 93, 062340 (2016).

[38] C. Lancien, S. Di Martino, M. Huber, M. Piani, G. Adesso, and A. Winter, Should Entanglement Measures be Monogamous or Faithful? Phys. Rev. Lett. 117, 060501 (2016).

[39] C. Eltschka, A. Osterloh, and J. Siewert, Possibility of generalized monogamy relations for multipartite entanglement beyond three qubits, Phys. Rev. A 80, 032313 (2009).

[40] C. Eltschka and J. Siewert, Distribution of entanglement and correlations in all finite dimensions, Quantum 2, 64 (2018).

[41] C. Eltschka, F. Huber, O. Gühne, and J. Siewert, Exponentially many entanglement and correlation constraints for multipartite quantum states, Phys. Rev. A 98, 052317 (2018).

[42] C. Eltschka and J. Siewert, Monogamy Equalities for Qubit Entanglement from Lorentz Invariance, Phys. Rev. Lett. 114, 140402 (2015).

[43] G. Gour and Y. Guo, Monogamy of entanglement without inequalities, Quantum 2, 81 (2018).

[44] N. Friis, G. Vitagliano, M. Malik, and M. Huber, Entanglement certification from theory to experiment, Nat. Rev. Phys. 1, 72 (2019).

[45] M. Genovese, Research on hidden variable theories: A review of recent progresses, Phys. Rep. 413, 319 (2005).

[46] G. H. Aguilar, A. Valdés-Hernández, L. Davidovich, S. P. Walborn, and P. H. Souto Ribeiro, Experimental Entanglement Redistribution under Decoherence Channels, Phys. Rev. Lett. 113, 240501 (2014).

[47] O. Jiménez Farías, A. Valdés-Hernández, G. H. Aguilar, P. H. Souto Ribeiro, S. P. Walborn, L. Davidovich, X.-F. Qian and J. H. Eberly, Experimental investigation of dynamical invariants in bipartite entanglement, Phys. Rev. A 85, 012314 (2012).

[48] S. Camalet, Monogamy Inequality for Any Local Quantum Resource and Entanglement, Phys. Rev. Lett. 119, 110503 (2017).

[49] S. Camalet, Simple state preparation for contextuality tests with few observables, Phys. Rev. A 94, 022106 (2016).

[50] S. Camalet, Monogamy inequality for entanglement and local contextuality, Phys. Rev. A 95, 062329 (2017).

[51] S. Camalet, Internal Entanglement and External Correlations of Any Form Limit Each Other, Phys. Rev. Lett. 121, 060504 (2018).

[52] M. Kuś and K. Życzkowski, Geometry of entangled states, Phys. Rev. A 63, 032307 (2001).

[53] F. Verstraete, K. Audenaert, and B. De Moor, Maximally entangled mixed states of two qubits, Phys. Rev. A 64, 012316 (2001).

[54] K. Życzkowski, P. Horodecki, A. Sanpera, and M. Lewenstein, Volume of the set of separable states, Phys. Rev. A 58, 883 (1998).

[55] G. Vidal and R. F. Werner, Computable measure of entanglement, Phys. Rev. A 65, 032314 (2002).

[56] V. Vedral and M. Plenio, Entanglement measures and purification procedures, Phys. Rev. A 57, 1619 (1998).

[57] P. G. Kwiat, E. Waks, A. G. White, I. Appelbaum, and P. H Eberhard, Ultrabright source of polarization-entangled photons, Phys. Rev. A 60, R773 (1999). 
[58] D. F. V. James, P. G. Kwiat, W. J. Munro, and A. G. White, Measurement of qubits, Phys. Rev. A 64, 052312 (2001).

[59] M. A. Nielsen and I. L. Chuang, Quantum Computation and Quantum Information (Cambridge University, Cambridge, England, 2000).
[60] H. S. Poh, S. K. Joshi, A. Cerè, A. Cabello, and C. Kurtsiefer, Approaching Tsirelson's Bound in a Photon Pair Experiment, Phys. Rev. Lett. 115, 180408 (2015).

[61] Z. Xi, Y. Li, and H. Fan, Quantum coherence and correlations in quantum system, Sci. Rep. 5, 10922 (2015). 